\section{Associations between perceived stress, quality of life and complementary health practices in Japanese outpatients: a multicenter observational study}

\author{
Tomoaki Kimura, ${ }^{1}$ Hiroshi Matsuo,1,2 \\ Naoharu lida, ${ }^{3}$ Yoshiteru Maki, 4 \\ Kiyoshi Suzuki1,5
}

1MOA Health Science Foundation, Shizuoka; ${ }^{2}$ Aiwakai Tatekawa Clinic, Osaka; ${ }^{3 Z u i s e n k a i ~ M O A ~ S a p p o r o ~ C l i n i c, ~}$ Hokkaido; ${ }^{4}$ Korinkai Kagoshima Clinic, Kagosima, 5 Gyokusenkai MOA Takanawa Clinic, Tokyo, Japan

\section{Abstract}

In Japan and other countries, complementary health practices based on lifestyle modification such as exercise, diet, art and culture, gardening, and biofield therapy, have received increasing attention. This study was conducted to demonstrate associations between perceived stress, quality of life (QOL), and these complementary health practices. Computer-assisted data collection was conducted with Japanese outpatients in 10 clinics. Participants completed questionnaires that measured socioeconomic status (SES) and frequencies of complementary health practices. Psychological stress was measured using the 10-Item Japanese version of the Perceived Stress Scale. Q0L was measured using the 10-Item Mokichi Okada Association Quality of Life Questionnaire. Data were analyzed using linear regression modeling. Baseline data of 1480 participants were available for cross-sectional analysis and data of 318 participants were available at follow-up for longitudinal analysis. Gender differences were not observed in stress and QOL, but age was positively correlated with QOL, and negatively with stress. A multiple regression model adjusted for age, gender, and SES indicated weak associations between each health practice and QOL in the cross-sectional $(\beta=0.14-0.28)$ and the longitudinal analysis $(\beta=0.17-0.27)$. Moreover, negative associations between each health practice and stress varied from -0.08 to -0.18 , with the exception of biofield therapy in the cross-sectional analysis. In the longitudinal analysis, associations varied from -0.13 to -0.27 , but diet and biofield therapy were not significantly associated with stress.

These results suggest that complementary health practices had a significant effect on maintaining, or improving $\mathrm{QOL}$, as well as in reducing stress of Japanese outpatients.

\section{Introduction}

Chronic illnesses such as lifestyle-related illness have increased in recent years. For preventing such illnesses and for their cure, physicians and other health providers encourage patients to modify their lifestyle, including diet and exercise, and to manage stress. ${ }^{1}$ Favorable lifestyles are known to be negatively associated with stress, ${ }^{2}$ and closely associated with good mental health. ${ }^{3}$ Most patients recognize the importance of lifestyle modification and stress management, but have difficulties in maintaining these practices. For example, it seems difficult to continue weight control programs. ${ }^{4}$ Interventions aimed at lifestyle modification conducted in general practice clinics have resulted in only small effects. ${ }^{5}$ Therefore, it is necessary to develop complementary health practices that are easy to conduct, feasible, and attractive.

The Mokichi Okada Association International (MOA) has developed and promoted the Okada Health and Wellness Program, ${ }^{6}$ which is a complementary health practice. The program is based on the philosophy and work of Mokichi Okada (1882-1955), a Japanese philosopher, and consists of three kinds of health practices: i) diet, ii) art and culture, and iii) biofield therapy. It has been empirically demonstrated that these practices are beneficial for maintaining and improving a patient's quality of life (QOL), for reducing stress, for providing motivation to modify the lifestyle, and thereby prevent diseases. Although these practices have come to be used widely in Japan and other countries, there is little reported evidence regarding their impact on improving Q0L or symptoms of illnesses. A number of studies have recently reported on the use of a type of biofield therapy known as Okada Purifying Therapy, in the general population, ${ }^{7}$ for menopausal symptoms of Japanese women, 8 for refractory migraine in Italian patients, ${ }^{9}$ for rheumatoid arthritis in Japanese patients, ${ }^{10}$ and regarding electroencephalographic effects. 11

The aim of this study was to demonstrate associations between perceived stress, Q0L, and complementary health practices in Japanese outpatients using linear regression modeling.

\section{Materials and Methods}

\section{Participants and procedure}

This study was conducted with the approval of the Institutional Review Board of the MOA Health Science Foundation, between April 2009 and June 2012 in ten clinics practicing
Correspondence: Tomoaki Kimura, MOA Health Science Foundation, 1-1-60 Nishi-Atami, Atami, Shizuoka 413-0038, Japan.

Tel. +81.557 .86 .0663 - Fax: +81.557 .86 .0665$

E-mail: t-kimura@mhs.or.jp

Key words: complementary health practice, multiple regression model, perceived stress, quality of life.

Acknowledgments: the authors are grateful to Dr. Kazuo Nitta and Dr. Kiyoshi Yamaoka for beneficial suggestions as well as to our colleagues: Dr. Seiya Uchida, Dr. Yasutami Tsuda, and Hiroyuki Sakaguchi for data collection. The authors also wish to express their sincere thanks to the participants of this study and medical doctors and staff of the clinics: MOA Sapporo Clinic of the Zuisenkai; MOA Takanawa Clinic, MOA Okuatami Clinic, MOA Nagoya Clinic, and Kanazawa Clinic of the Gyokusenkai; Tatekawa Clinic, Hiroshima Clinic, and MOA Kochi Clinic of the Aiwakai; Kagoshima Clinic and Okinawa Clinic of the Korinkai.

Contributions: TK, data analysis and interpretation of data, and completed this manuscript; HM, $\mathrm{KS}$, design of this survey and data collection; NI, YM, data collection.

Conflict of interests: the authors declare no potential conflict of interests.

Funding: this study was supported within a budget of the MOA Health Science Foundation, nonprofit organization.

Received for publication: 31 March 2013

Revision received: 18 May 2013.

Accepted for publication: 18 May 2013

This work is licensed under a Creative Commons Attribution NonCommercial 3.0 License (CC BYNC 3.0).

(C) Copyright T. Kimura et al., 2013

Licensee PAGEPress, Italy

Alternative Medicine Studies 2013; 3:e1

doi:10.4081/ams.2013.e1

integrated medicine. In the clinics physicians treated diseases and cared for patients together with practitioners of complementary medicine. These clinics were located from the northern to the southern end of Japan (Figure 1). Before commencing the study, its purpose and procedure was explained to physicians and other staff of the clinics by one of the authors and his colleagues. A tablet personal computer (PC) with a touch screen monitor was setup to collect questionnaire data. Physicians or welltrained paramedical staff asked a portion of outpatients over 20 years of age to participate in this study, and informed the patients of the aims of the survey using an information sheet. 
The information sheet indicated: i) participants should state the facts as they are, but could skip a question if they did not like to respond; ii) they would receive neither special favors nor an honorarium for participation; iii) if they declined to participate or withdraw, they would not be penalized in any way; and iv) data would be strictly guarded and personal information would not be disclosed. After the participants understood these explanations, they signed the informed consent form. Then the physicians classified the patients' illnesses based on their diagnoses, and input the data into the PC. Participants completed questionnaires that assessed their socioeconomic status (SES), frequencies of complementary health practices, perceived stress, and generic QOL that were presented on the tablet PC screen. Some of the participants completed the questionnaires again after several months at a follow-up assessment.

\section{Questionnaires}

Computer-assisted data collection was conducted using a series of questionnaires.

One of the authors (TK) and his colleagues have used PC version of the questionnaires and confirmed its accessibility, reliability and validity.12,13 SES question items comprised annual income, occupation, education, and marital status. Question items assessing five complementary health practices comprised exercise, diet, art and culture, gardening, and biofield therapy.

Psychological stress was measured using the 10-Item Japanese version of the Perceived Stress Scale (JPSS-10). The JPSS was translated from the Perceived Stress Scale, 14 a 14-item self-administered instrument, by Iwahashi and his colleagues. ${ }^{15} \mathrm{~A}$ brief version of the JPSS consisting 10 items designed to reduce respondents' burden and save time has been developed. ${ }^{16}$ Responses to the JPSS-10 are made on a five-point Likert-type scale; with 4 of the 10 items being reverse scoring questions. Each item is scored from 0 to 4 , such that the range of the total score varies from 0 to 40 , with a higher score indicating a greater perception of stress.

Generic QOL was measured using the 10 Item MOA Quality of Life Questionnaire (MQL10). The MQL-10 has been developed by the authors to assess QOL and determine the effects of health practices in large-scale health surveys. 17 The MQL-10 is a Likert-type questionnaire consisting of 10 items that are scored on a 5-point scale. The MQL-10 is designed to assess physical, mental and social wellbeing. Each item is scored from 0 to 4 ; therefore, the range of the total score is from 0 to 40 , with higher scores indicating better QOL. The validity of this measure has been established through comparison with the short version of the World Health Organization
Quality of Life instrument (WHOQOL-BREF, 26 items) ${ }^{18}$ and the 36 -Item Short Form Health Survey (SF-36), ${ }^{19}$ which are used globally. This validation study had demonstrated that the correlation coefficient between the total score of the MQL-10 and the average score of the WHOQOL-BREF was $0.81(\mathrm{P}<0.001)$; and the correlations with the general health perception, vitality, and mental health domains of the SF-36 were $0.58,0.62$, and 0.64 , respectively $(\mathrm{P}<0.001) .17$ The internal consistency reliability was determined with the Cronbach's alpha coefficient (0.85). 17 The responsiveness of this measure has also been established by a longitudinal study. ${ }^{20}$

\section{Complementary health practices}

Physicians and staff of the clinics collaborating with the study recommended complementary health practices to the participants that were followed on a voluntary basis. ${ }^{6}$ The complementary health practices included 5 items: i) diet, ii) exercise, iii) gardening, iv) art and culture, and v) biofield therapy.

The recommended health practice for diet was to eat fresh, seasonal products, such as organic vegetables, among others and to exercise and/or do gardening. The art and culture recommendation was to enjoy the arts, music, traditional culture and natural beauty, among others. Biofield therapy was also recommended; this therapy is an energy therapy (treatment without touching), called Okada Purifying Therapy (OPT), ${ }^{21,22}$ or Johrei, $23-25$ which was originally developed by Mokichi Okada. Its purpose is to maintain health and improve symptoms related to daily life. OPT can be practiced only by licensed practitioners that have completed a training program for OPT treatment as an alternative therapy. Since these recommendations were not compulsory intervention programs, a questionnaire was used to determine the frequency or the level of participants' health practices during the past three months. For example, question of exercise was how often did you exercise?, and the response choices were every day, almost every day, sometimes, rarely, and not at all. In diet, the patients were asked how much did you have natural or organic products?, and the response choices were over $80 \%, 60-80 \%, 40$ $60 \%, 20-40 \%$, and under $20 \%$.

\section{Statistical analysis}

A cross-sectional analysis was conducted to examine the associations of gender, age, SES, and complementary health practices with QOL and stress. First, a single linear regression analysis was conducted. Either QOL (MQL-10 score) or stress (JPSS-10 score) was set as a dependent variable with gender, age, SES, and complementary health practices as the explanatory (independent) variables. Sum of the frequencies of the five complementary health practices was also used as a variable in the analysis. Among SES, occupation was divided into two categories: i) employed or self-employed, and ii) unemployed, which included homemakers and students. Education was also divided into two categories: i) not more than 13 years, including high school, ii) more than 12 years. Marital status included: i) married, and ii) unmarried, which also included widowed and divorced people. Second, a multiple linear regression analysis was conducted. Either Q0L (MQL-10 score), or stress (JPSS-10 score) was set as a dependent variable with gender, age, SES, and one of the complementary health practices as explanatory variables (forced entry). Among the SES, annual income was excluded from the multiple regression model, because it was not significant, and had a number of missing data points.

A longitudinal analysis was also conducted to determine the associations of health practices with QOL and stress at follow-up assessment. Mean values of the frequencies of complementary health practices at baseline and follow-up were used in the analysis and single and multiple linear regression analyses were conducted. Statistical analyses were conducted using SPSS Statistics, version 20 (IBM Corporation) with the statistical significance level set at $\mathrm{P}<0.05$.

Table 1. Classification of disease of participants (the classification allowed multiple responses).

\begin{tabular}{lcc} 
Disease & Frequency & $\%$ \\
Chronic disease & 1190 & 80.4 \\
Malignant neoplasm & 30 & 2.0 \\
MN after surgery & 51 & 3.4 \\
Benign neoplasm & 21 & 1.4 \\
BN after surgery & 8 & 0.5 \\
Anemia & 74 & 5.0 \\
Endocrine disease & 40 & 2.7 \\
Diabetes & 104 & 7.0 \\
Obesity & 42 & 2.8 \\
Dyslipidemia & 262 & 17.7 \\
Psychiatric disease & 7 & 0.5 \\
Neurotic disease & 38 & 2.6 \\
Cranial nerve disease & 15 & 1.0 \\
Allergic disease & 54 & 3.6 \\
Heart disease & 70 & 4.7 \\
Hypertension & 287 & 19.4 \\
Cerebrovascular disease & 35 & 2.4 \\
Respiratory disease & 46 & 3.1 \\
Gastrointestinal disease & 43 & 2.9 \\
Liver or pancreatic disease & 79 & 5.3 \\
Arthritis/gout & 67 & 4.5 \\
Rheumatic/ & 34 & 2.3 \\
Connective tissue disease & & \\
Renal disease & 31 & 2.1 \\
Others & 120 & 8.1 \\
\hline Acute or infectious disease & 23 & 1.6 \\
Injury or fracture & 10 & 0.7 \\
\hline Subjective symptom only & 172 & 11.6 \\
\hline
\end{tabular}




\section{Results}

Baseline data of 1480 participants (379 men and 1101 women; mean age 58.5) were available for the cross-sectional analysis. The number of participants in each clinic is shown in Figure 1. Of the 1480 participants, 318 (99 men and 219 women; mean age 56.8 ) were available for the longitudinal analysis at the follow-up. Mean interval between baseline and follow-up was 9.4 months.

Table 1 indicates the illness classification of participants based on the physicians' diagnosis. Comorbid diseases were also classified; and therefore multiple responses are shown. Most outpatients had chronic illnesses (80.4\%) such as hypertension (19.4\%), dyslipidemia (17.7\%), and diabetes (7.0\%).

SES of the participants is displayed in Table 2. Of the participants, $38.8 \%$ were employed; when self-employed people were included, this number increased to $45.5 \%$. The mode for their annual income was 2-5 million Yen (40.4\%), but there were a number of missing data points in this item. The mode of education was high school graduation (53.1\%). Moreover, $72.4 \%$ of the participants were married.

Results of a single regression analysis with QOL and stress as dependent variables were shown in Table 3. The associations between explanatory variables and dependent variables were illustrated with standardized regression coefficients $(\beta)$. In the cross-sectional analysis, gender differences were not observed for stress and Q0L, but age was positively correlated with QOL and negatively with stress. Among SES, employed and highly-educated participants had more stress; married participants had less stress, but annual income was not significantly associated with QOL or stress. Moreover, the weak associations between the frequency of each complementary health practice and QOL were indicated in the cross-sectional analysis $(\beta=0.14-0.27)$ and in the longitudinal analysis $(\beta=0.15-0.28)$. Negative associations between the frequency of each health practice and stress varied from -0.06 to -0.23 in the cross-sectional analysis. Diet and biofield therapy were not significantly associated with stress in the longitudinal analysis. The sum of frequencies of the five complementary health practices was more closely associated with QOL. Results of a multiple regression analysis for QOL and stress are shown in Table 4. There were weak associations between the frequency of each health practice and QOL in the crosssectional $(\beta=0.14-0.28)$ and the longitudinal analysis $(\beta=0.17-0.27)$. Negative associations between the frequency of each health practice and stress varied from -0.08 to -0.18 , with the exception of biofield therapy in the cross-sec-
Table 2. Socioeconomic status of participants.

\begin{tabular}{llcc}
\hline Item & Category & Frequency & $\%$ \\
Occupation & Employed & 574 & 38.8 \\
& Self-employed & 99 & 6.7 \\
& Homemaker & 566 & 38.2 \\
& Student & 7 & 0.5 \\
& Unemployed & 230 & 15.5 \\
Annual income* & Missing & 4 & 0.3 \\
& $<2$ Million Yen & 426 & 28.8 \\
& $2-5$ Million Yen & 598 & 40.4 \\
& 5-7 Million Yen & 147 & 9.9 \\
& $7-10$ Million Yen & 65 & 4.4 \\
& $\geq 10$ Million Yen & 28 & 1.9 \\
& Missing & 216 & 14.6 \\
\hline Education & $\leq 9$ Years & 220 & 14.9 \\
& High School & 786 & 53.1 \\
& College & 156 & 10.5 \\
& Vocational School & 163 & 11.0 \\
& University & 147 & 9.9 \\
& Missing & 8 & 0.5 \\
Marital status & Married & 1072 & 72.4 \\
& Widowed & 175 & 11.8 \\
& Divorced & 58 & 3.9 \\
& Unmarried & 172 & 11.6 \\
& Missing & 3 & 0.2 \\
\hline
\end{tabular}

1 Million Yen = 12,500 US Dollars (2012).

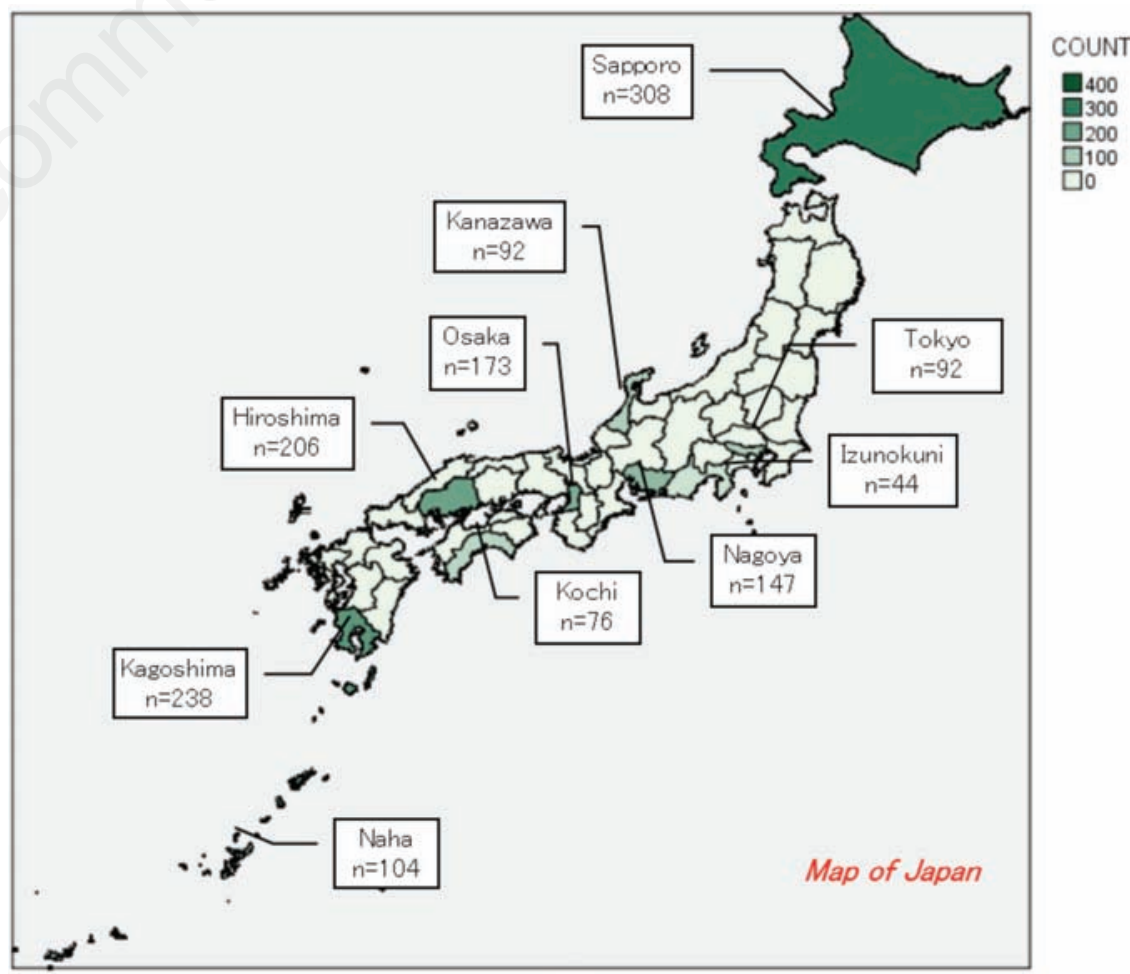

Figure 1. Distribution of clinics and subjects of this study. The total number of subjects was 1480 . 
tional analysis. In the longitudinal analysis, associations varied from -0.13 to -0.27 , but diet and biofield therapy were not significantly associated with stress. The sum of frequencies of the five complementary health practices was also more closely associated with both QOL and stress, even after being adjusted for gender, age, and SES.

\section{Discussion}

The results of this study demonstrated a number of significant regression models, the outlines of which are as follows. Complementary health practices were associated positively with QOL and negatively with stress both in the cross-sectional and the longitudinal analyses. Among complementary health practices, exercise was most closely associated with QOL in the longitudinal analysis. Art and culture practice was most negatively associated with stress in the longitudinal analysis. Multiple regression models adjusted for age, gender, and SES corresponded with the above results.

Associations between complementary health practices and QOL corresponded to a previous study regarding the responsiveness of the MQL-10,20 which was conducted using a population of healthy adults without SES data. The result of the present study extended the results of the previous study, because we used a sample of outpatients with chronic illnesses and the data adjusted by SES. In the current study, the sum of frequencies of the five health practices was more closely associated with QOL. This result suggests that a combination of health practices might be more effective. The result that exercise was most closely associated with QOL might be related to the fact that good physical functioning is required to do exercise and good physical functioning also increases the QOL. Exercise is considered effective for stress management as well as in physical activities. The result of this study corroborated findings on the benefit of exercise that has been suggested by previous studies.

Negative associations between complementary health practices and stress were a new finding. It has been empirically demonstrated that these health practices have psychological effects. It is well known that moderate exercise, or hobby activities reduce psychological stress. For example, Nakanishi and her colleagues demonstrated that horticultural activities reduced stress among patients with diabetes, ${ }^{26}$ but this study was conducted as a case series study and the findings of the present study offer evidence with more validity. Art and culture practice includes appreciation of the beauty of nature, flower, and arts. Ulrich et $a l .{ }^{27}$ has described the theory and provided evi- dences on the stress reducing effects of nature. Weil, a pioneer of integrative medicine, ${ }^{28}$ also recommended art, flowers, and animals for emotional and spiritual wellbeing. Otsuka et al. ${ }^{29}$ reported that flowers and tea therapy, which is one of the art and culture practices, improved the symptoms and psychological conditions in rheumatoid arthritis patients. Art and culture activities are different by ethnicity or country. In some countries, music or dance may be effective for stress reduction. Further comparative studies need to be conducted to examine the cultural differences and adaptation.

In the longitudinal analysis, diet was associated with QOL, but not with stress. Generally, diet therapy is based on the management of nutrition and calories, and might be a stressor. The diet practice of MOA, however, is focused on the freshness and the inherent spirit of food, as well as gratitude toward food and food producers. Therefore, diet practice was negatively associated with stress in the cross-sectional analysis, and in the longitudinal analy-

Table 3. Results of single regression analyses for quality of life (QOL) and stress as dependent variables.

\begin{tabular}{|c|c|c|c|c|}
\hline \multirow[t]{2}{*}{ Explanatory variable } & \multicolumn{2}{|c|}{ Cross-sectional analysis } & \multicolumn{2}{|c|}{ Longitudinal analysis } \\
\hline & QOL & Stress & QOL & Stress \\
\hline \multicolumn{5}{|c|}{$\begin{array}{l}\text { Frequency of complementary } \\
\text { health practice }\end{array}$} \\
\hline Exercise & $0.241^{*}$ & $-0.213^{*}$ & $0.279 *$ & $-0.246^{*}$ \\
\hline Diet & $0.266^{*}$ & $-0.169^{*}$ & $0.217^{*}$ & ns \\
\hline Art and culture & $0.272^{*}$ & $-0.227^{*}$ & $0.148 * *$ & $-0.369^{*}$ \\
\hline Gardening & $0.171^{*}$ & $-0.189 *$ & $0.176^{* *}$ & $-0.294^{*}$ \\
\hline Biofield therapy & $0.139^{*}$ & $-0.062 * * *$ & $0.203^{*}$ & ns \\
\hline Sum of frequencies & $0.351^{*}$ & $-0.277^{*}$ & $0.350^{*}$ & $-0.318^{*}$ \\
\hline Age & $0.169 *$ & $-0.354^{*}$ & ns & $-0.405^{*}$ \\
\hline Occupationa & ns & $0.156^{*}$ & ns & $0.296 *$ \\
\hline Education $^{\mathrm{b}}$ & ns & $0.088^{*}$ & ns & $0.159^{* *}$ \\
\hline Marital statusc & ns & $-0.067 * * *$ & ns & ns \\
\hline
\end{tabular}

Cross-sectional analysis $(\mathrm{n}=1480)$ and longitudinal analysis $(\mathrm{n}=318$, mean interval 9.4 months) were conducted using single regression analysis. Standardized coefficients $(\beta)$ were indicated. Gender and annual income were not significant (not shown). ${ }^{*} \mathrm{P}<0.001 ;{ }^{* *} \mathrm{P}<0.01$; ${ }^{* * *}<0.05$ $\mathrm{ns}$, not significant. aOccupation was divided into employed and self-employed (1) and unemployed (0); bEducation was divided into under 10 years or high school (0) and over 12 years (1); cMarital status was divided into marriage (1) and others (0).

Table 4. Results of multiple regression analyses for quality of life (QOL) and stress as dependent variables.

$\begin{aligned} & \text { Explanatory variablea } \\ & \text { Frequency }\end{aligned}$
$\begin{aligned} & \text { Co complementary } \\ & \text { of comectional analysis }\end{aligned}$
health practice

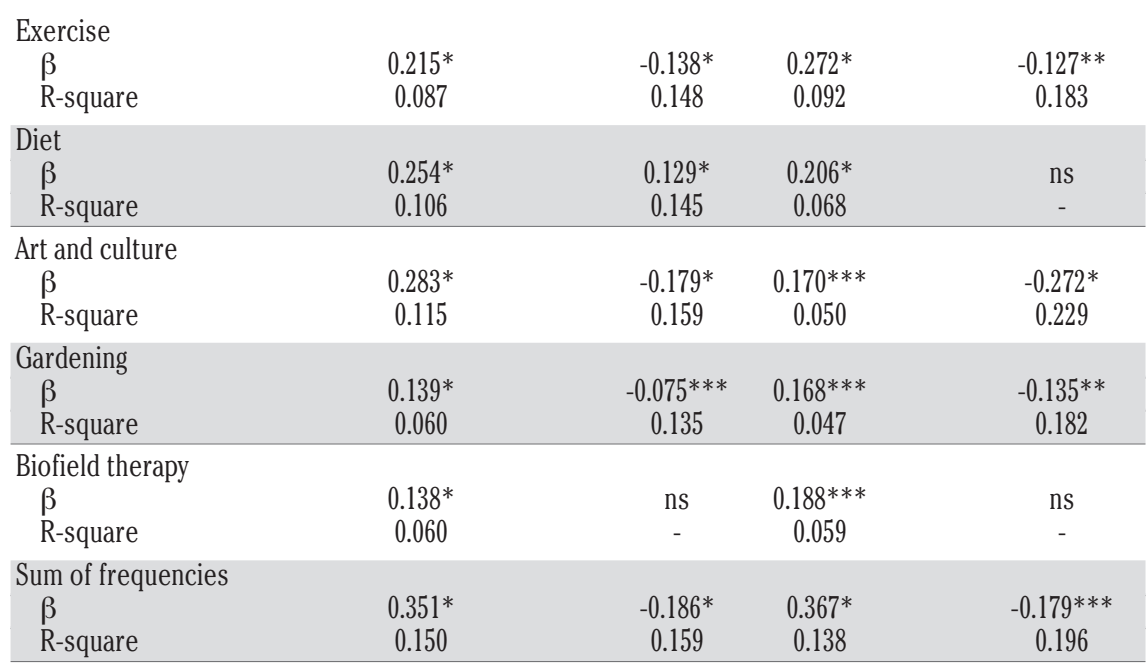

Cross-sectional analysis $(n=1480)$ and longitudinal analysis $(n=318$, mean interval 9.4 months) were conducted using multiple regression analysis (forced entry). Standardized coefficients $(\beta)$ and R-square statistics of the models were indicated. aGender, age, occupation, education, and marital status were used as explanatory variables. ${ }^{*} \mathrm{P}<0.001 ;{ }^{*} \mathrm{P}<0.05 * * * \mathrm{P}<0.01$; ns, not significant. 
sis it was not associated with stress. Since increasing attention has been given to dietary intervention in both lifestyle modification and complementary medicine, it is suggested that more specific studies be conducted regarding this topic.

In the longitudinal analysis, biofield therapy, as well as other health practices was associated with QOL, but not with stress. A previous study has reported that the effects of biofield therapy on QOL were relatively small, ${ }^{20}$ because participants with current illnesses that had lower QOL scores tended to practice it frequently. In the present study that was conducted in a clinical setting, presumably, there was a counteracting effect of healthy participants with higher QOL scores. Previous studies on the effects of biofield therapy that have conducted pre and post biofield therapy comparisons have also clearly demonstrated that OPT improved shoulder stiffness and reduced stress, ${ }^{30}$ and that Johrei improved negative emotional states. ${ }^{24}$

Biofield therapy is also considered to be a relaxation practice. ${ }^{23,30}$ Dusek et al. ${ }^{31}$ reported that relaxation was more effective for hypertension than a lifestyle modification program. Motivational readiness and compliance with lifestyle modification are key issues. The combination of complementary health practices including lifestyle modification and stress management may be more effective than an intervention using a single practice. The result of this study that the sum of frequencies of the five complementary health practices was more closely associated with both QOL and stress suggests that the additional or synergistic effect may be more important than an effect of individual practices. For example, OPT may have supported motivation and compliance with other health practices. In this sample, it is impossible to distinguish between initial consultation and others. Therefore, it was a mixture of patients who had experienced OPT and other practices, and those who began health practices after the initial visit. If the beginners could be chosen, more drastic effects might have been shown. Further studies need to be conducted in order to clarify these hypotheses.

This study, however, has several limitations. One is that random sampling was not used. Although the participants were distributed throughout Japan, the ratio of samples by area did not correspond to the population distribution (Figure 1). Secondly, the gender and age of the participants were biased with more women participating than men. However, the results of multiple regression models adjusted for gender and age were similar to the results of the single regression models. Thirdly, the illness of the participants varied widely. Therefore, the results and interpretations of this study might differ according to the type, or the severity of diseases. Lastly, this study was neither an intervention study nor a clinical trial, but an observational study. In the longitudinal analysis, associations between past frequencies of health practices and present QOL or stress were examined, but efficacy of the health practices could not be substantially established. Regardless of these limitations, we consider that this study partially demonstrated the associations between Q0L, stress, and complementary health practices in Japanese outpatients. It is concluded that the associations indicated in this study suggest that the complementary health practices might have a significant effect on maintaining, or improving $\mathrm{QOL}$, as well as in reducing the stress level of outpatients. To verify these findings, a clinical trial, or a long-term prospective study investigating certain illnesses should be conducted in the future.

\section{References}

1. Marmot M, Wilkinson RG, eds. Social determinants of health. Oxford, UK: Oxford University Press; 1999.

2. Ezoe S, Morimoto K. Behavioral lifestyle and mental health status of Japanese factory workers. Prev Med 1994;23:98-105.

3. Suda M, Nakayama K, Morimoto K. Relationship between behavioral lifestyle and mental health status evaluated using the GHQ-28 and SDS questionnaires in Japanese factory workers. Industrial Health 2007;45:467-73.

4. Cresci B, Rotella CM. Motivational readiness to change in lifestyle modification programs. Eat Weight Disord 2009;14:15862.

5. Ashenden R, Silagy C, Weller D. A systematic review of the effectiveness of promoting lifestyle change in general practice. Fam Pract 1997;14:160-76.

6. MOA International Corporation. Okada Health and Wellness Program. Available from: http://www.moainternational. or.jp/en/health/therapy.html. Accessed on: May 14, 2013.

7. Suzuki K, Uchida S, Kimura T, Katamura H. A large cross-sectional descriptive study of self-reports after biofield therapy in Japan: demography, symptomatology, and circumstances of treatment administration. Altern Ther Health Med 2012;18:3550 .

8. Hatayama M, Suzuki K, Ishida A, et al. Long-term efficacy of Okada purifying therapy (bio-energy healing) on menopausal symptoms. Res Rep MOA Health Sci 2009;12:5-12.

9. Bruti G, Ramos M. Okada purifying therapy in refractory migraine: a pilot study. Res Rep MOA Health Sci 2010;14:5-15.
10. Otsuka M, Takeda M, Kuriki S, et al. A study on the effects of three months continued treatments of Okada Purifying Therapy in rheumatoid arthritis patients. Soc Integr Med Jpn 2012;5:66-72.

11. Uchida S, Iha T, Yamaoka K, et al. Effect of biofield therapy in the human brain. $\mathrm{J}$ Altern Complement Med 2012;18:875-9.

12. Kimura T, Uchida S, Tsuda Y, Eboshida A. Computer-assisted measurement of perceived stress: an application for a community-based survey. Hiroshima J Med Sci 2005;54:61-5.

13. Kimura T, Tsuda Y, Uchida S, et al. Validity of computer-assisted health assessment using visual analogue scales and face scales: a comparison between younger and older participants. Jpn J Appl Psychol 2012; 38:101-8.

14. Cohen S, Kamarck T, Mermelstein R. A global measure of perceived stress. J Health Soc Behav 1983;24:385-96.

15. Iwahashi S, Tanaka Y, Fukudo S, Hongo M. The development of the Japanese version of the Perceived Stress Scale. Jpn J Psychosom Med 2002;42:459-66.

16. Kimura T, Tsuda Y, Uchida S, et al. Development of the shortened questionnaires for the Japanese version of the Perceived Stress Scale. Res Rep MOA Health Sci 2009;12:21-5.

17. Kimura T, Suzuki K, Morioka H, et al. Development and validation of the 10-Item MOA Quality of Life Questionnaire (MQL10) for large-scale health survey. Res Rep MOA Health Sci 2009;13:73-84.

18. Management of Substance Abuse, World Health Organization. WHO Quality of LifeBREF (WHOQOL-BREF). Available from: http://www.who.int/substance_abuse/rese arch_tools/whoqolbref/en/. Accessed on: May 14, 2013.

19. Ware JE, Sherbourne C. The MOS 36-item short-form health survey (SF-36): I. Conceptual framework and item selection. Med Care 1992;30:473-83.

20. Kimura T, Suzuki K, Uchida S, Katamura S. Responsiveness and minimally important difference of a generic quality of life measure for complementary health practices. Altern Med Studies 2012;2:e12.

21. Nitta K. Complementary and alternative medicine. Biotherapy 2005;19:167-79.

22. Suzuki K, Nitta K, Katamura H. Clinical application of bio-energy medicine in interrative medicine: from background to clinical practice. Biotherapy 2007;21:40713.

23. Naito A, Laidlaw TM, Henderson DC, et al. The impact of self-hypnosis and Johrei on lymphocyte subpopulations at exam time: a controlled study. Brain Res Bull 2003; 62:241-53.

24. Reece K, Schwartz GE, Brooks AJ, Nangle 
G. Positive well-being changes associated with giving and receiving Johrei healing. J Altern Complement Med 2005;11:455-7.

25. Canter PH, Brown LB, Greaves C, Ernst E. Johrei family healing: a pilot study. Evid Based Complement Alternat Med 2006;3: 533-40.

26. Nakanishi Y, Sakai A, Morioka H, Shibata K. Application and evaluation of horticultural activities for patients with diabetes. Res Rep MOA Health Sci 2010;14:29-42.

27. Ulrich RS, Simons RF, Losito BD, et al.
Stress recovery during exposure to nature and urban environments. J Environ Psychol 1991;11:201-30.

28. Weil A. Secular spirituality and emotional well-being. In: Spontaneous happiness. New York: Little, Brown and Company; 2011. pp 167-194.

29. Otsuka M, Kuriki S, Noda $\mathrm{H}$, et al. Influence in disease activity and cytokines of health and wellness program using the flower therapy and the Japanese tea therapy in rheumatoid arthritis patients. Res
Rep MOA Health Sci 2010;14:17-27.

30. Uchida S, Tsuda Y, Kimura T, et al. Assessing perceived shoulder stiffness using hardness meters. Jpn J Psychosom Med 2011;51:1120-32.

31. Dusek JA, Hibberd PL, Buczynski B, et al. Stress management versus lifestyle modification on systolic hypertension and medication elimination: a randomized trial. $\mathrm{J}$ Altern Complement Med 2008;14:129-38. 